\title{
The role of the transition state in muon implantation
}

\author{
R C Vilão,, , $\mathrm{R}$ B L Vieira, ${ }^{1} \mathrm{H}$ V Alberto, ${ }^{1} \mathrm{~J}$ M Gil, ${ }^{1}$ and A Weidinger ${ }^{2}$ \\ ${ }^{1}$ CFisUC, Department of Physics, University of Coimbra, P-3004-516 Coimbra, Portugal \\ ${ }^{2}$ Helmholtz-Zentrum Berlin für Materialien und Energie, 14109 Berlin, Germany
}

(Dated: September 28, 2017)

\begin{abstract}
In muon-spin-rotation experiments, positive muons are implanted in the material and come to rest in the unrelaxed host lattice. The formation of the final configuration requires a lattice relaxation which does not occur instantly. The present paper is concerned with the transition from the initial stopping state to the final muon configuration. We identify the often observed fast relaxing signal in muon experiments (e.g. in several oxides studied recently) with the transition state in this conversion process. This state is paramagnetic with a small hyperfine interaction (in the order of $\mathrm{MHz}$ ) which fluctuates and averages to almost zero. Because of its apparent diamagnetic frequency behavior, the fast signal was in the past assigned to $\mathrm{Mu}^{+}$or $\mathrm{Mu}^{-}$. We present evidence that this state is actually paramagnetic. The model presented in this paper is of importance for the interpretation of past and future $\mu \mathrm{SR}$ measurements.
\end{abstract}

PACS numbers: 76.75.+i, 71.55.-i, 61.72.-y, 71.38.-k

\section{INTRODUCTION}

In condensed mater positive muons behave similarly to hydrogen and are often used in materials research as local probes and substitutes for hydrogen. ${ }^{1 / 2}$ In muon spectroscopy experiments muons are implanted in the material and come to rest at a site which may not be the final position. The transition of the stopped muon from the initial to the final configuration is the subject of this paper. In some cases there is a barrier in this conversion process which leads to delay in the formation of the final states.

The slowing-down of the muon proceeds via different processes $\stackrel{3}{3}$ at high velocities, the impact ionization of the matrix atoms is dominant. When the velocity of the muon becomes comparable to the velocity of the matrix electrons, cyclic charge exchange between positive to neutral sets in. This process ends when the threshold for electron pickup and electron loss is reached. In semiconductors, at this stage the muon has energies of the order of $\mathrm{eV}$ and the charge state is positive or neutral. The negative charge state is unlikely since it is formed by a two-step process and the binding of the second electron is weak.

The remaining kinetic energy (eV region) after the charge exchange cycle is lost by excitation of phonons. The muon finally stops at an interstitial site in the yet unrelaxed lattice. The final configurations after the lattice relaxation are $!^{\sqrt[145]{45}} \mathrm{i}$ ) atom-like muonium at an interstitial site (e.g. normal muonium in $\mathrm{Si}$ ), ii) neutral muonium bound into the lattice structure (e.g. bond-centered muonium in Si), and iii) the positively charged state $\mathrm{Mu}^{+}$, also bound into the host lattice. In the present paper we are in particular concerned with the formation of these final states, starting from neutral muonium at an interstitial site in the unrelaxed lattice.

As an example of the present model, we will discuss the fast relaxing signal observed in several experiments, for example in oxides (Fig. 1). We assign this signal to the transition state which exists for some time during the conversion from the initial to the final muon configuration. A characteristic of this signal is that the precession frequency corresponds closely to the Larmor frequency of the diamagnetic muon. We will call it diamagnetic-like leaving open that the actual configuration of the muon may possibly differ from diamagnetic.

As an example, we now analyze the data of the slightly Mg-doped zirconia sample $\left(\mathrm{Zr}_{0.96} \mathrm{Mg}_{0.04} \mathrm{O}_{2}\right)$. The reason for this choice is that more data are available for this sample than for other systems. However, the features discussed here are typical for all zirconia (doped and undoped $\sqrt{6}$ and are also representative for other systems with a fast relaxing diamagnetic-like signal (see below).

The classic case of the fast relaxing signal is $\mathrm{Al}_{2} \mathrm{O}_{3}$ (Refs. 7[-9). This signal was first interpreted as being due to delayed capture by $\mathrm{Mu}^{+}$of an electron from the ionization track (delayed muonium formation, see Ref. 8). Later, electric-field measurements showed that this interpretation could not be confirmed and the signal was then assigned to initially formed $\mathrm{Mu}^{-}$which loses the electron after some time ${ }^{9}$ Cox et al ${ }^{10}$ mention that neither of the two assignments is satisfactory (the sapphire puzzle), $\mathrm{Mu}^{-}$formation being not very plausible.

Fast relaxing diamagnetic-like signals were observed also in various other systems: e.g. I. Fan et al. in photo-excited Ge, $\frac{11}{11}$ H. V. Alberto et al. in solar cell materials, $\frac{12}{12}$ R. B. L. Vieira et al. in $\mathrm{HfO}_{2},{ }^{13}$ R. C. Vilão in $\mathrm{TeO}_{2}, 14$

\section{USUAL INTERPRETATION OF THE FAST RELAXING SIGNAL}

The fast relaxing signal (Fig. 1) shows a precession frequency which is similar to the frequency of the diamagnetic state and has therefore been assigned to either $\mathrm{Mu}^{+}$or $\mathrm{Mu}^{-}$(for example Refs. 791315). It was, however, not tested whether the state is really diamagnetic 

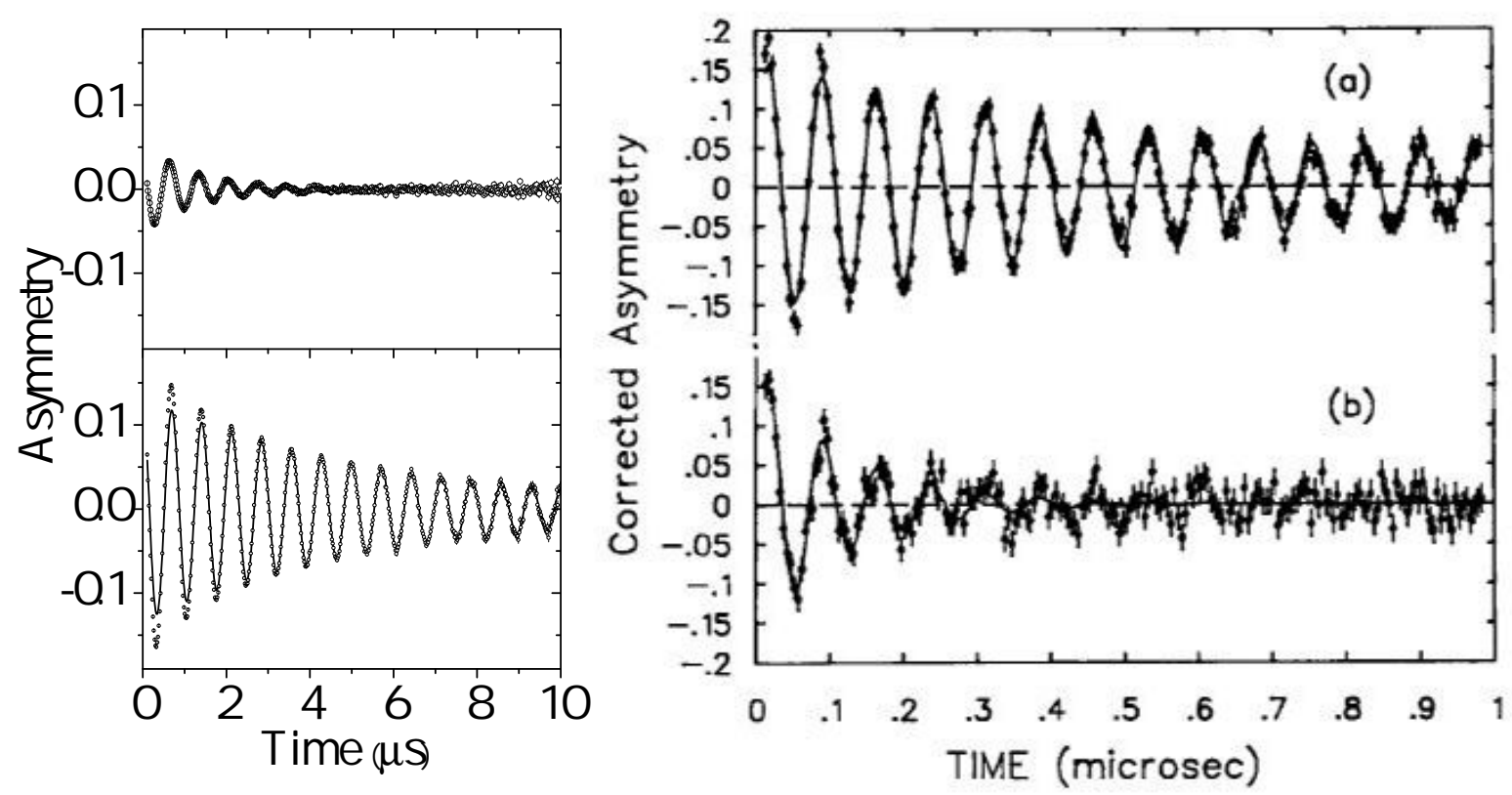

FIG. 1: Left: $\mu \mathrm{SR}$ signal of slightly Mg-doped zirconia $\mathrm{ZrO}_{2}: \mathrm{Mg}$ at $8 \mathrm{~K}$ (data from Ref. 6). The signal (full data in the lower frame) is decomposed in a slowly relaxing component (solid line in the lower frame) and a fast relaxing component (upper part of the figure). Right: $\mu \mathrm{SR}$ spectra of $\mathrm{Al}_{2} \mathrm{O}_{3}$ at (a) 8(2) $\mathrm{K}$ and (b) 16(3) $\mathrm{K}$ (figure from Ref. 7, copied with permission). The solid lines are fit curves with a single exponential function but with different relaxations. The precession frequencies in all cases are close to the Larmor frequency of diamagnetic muon.

or is actually paramagnetic with an average hyperfine interaction so small that the hyperfine splitting cannot be observed. A comparison of transverse and longitudinal field data (at appropriate fields) could clarify this point. The presence of a frequency shift would confirm as well the paramagnetic character. But these effects are small and not always detectable experimentally. We will come back to this point later but discuss first some shortcomings of the $\mathrm{Mu}^{+}$or $\mathrm{Mu}^{-}$interpretation.

\section{1. $\mathrm{Mu}^{+}$hypothesis}

One assumes that the muon stops as $\mathrm{Mu}^{+}$and exists for some time (several ns and more) in this charge state before it captures an electron from the ionization track (e.g. Ref. 15). There are several shortcomings in this interpretation: i) $\mathrm{Mu}^{+}$is not stable at the interstitial site and would quickly form the very stable $\mathrm{O}-\mathrm{H}$ like bond from which a return, after electron capture, to the higher-energy interstitial configuration is very unlikely. ii) If both fast and slowly relaxing signals correspond to $\mathrm{Mu}^{+}$, there is no place for the case where the muon stops in the neutral charge state. But it is unlikely that in the present cases no neutral muonium is formed in the charge exchange process.

The delayed capture of an electron from the ionization track was used to derive the electron mobility via the time the electron needs to travel from the ionization region to the muon site (Ref. 8). This measuring method is not applicable if the electron is already present near the muon from the stopping process, as proposed in the present work.

\section{2. $M u^{-}$hypothesis}

The formation of $\mathrm{Mu}^{-}$is a two-step process and as such strongly suppressed. Note that $\mathrm{Mu}^{-}$would have to be formed promptly since otherwise dephasing would destroy the polarization. The $\mathrm{Mu}^{-}$in question here (probably atom-like interstitial $\mathrm{Mu}^{-}$) is not the strongly bound hydride-like negative muonium predicted by theory (e.g. Ref. 16) since the formation of this latter configuration requires a large lattice rearrangement which is unlikely in the short time and the relatively low temperature of the muon experiment. On the other hand, the atom-like negative muonium, the analogue of the quasi-free $\mathrm{H}^{-}$, is very unstable if embedded in the host lattice. It is conceivable that this $\mathrm{Mu}^{-}$is formed for a short time in a fluctuating capture and loss process as reported in the experiment on highly-doped n-type samples at high temperatures, $\frac{17}{, 7}$ or possibly in n-type samples after the ionization of donors, again in a cyclic capture and loss proces $\$^{18 / 19}$ though in the latter case a revision of the assignment is intended following this work. However, a $\mathrm{Mu}^{-}$with the charac- 


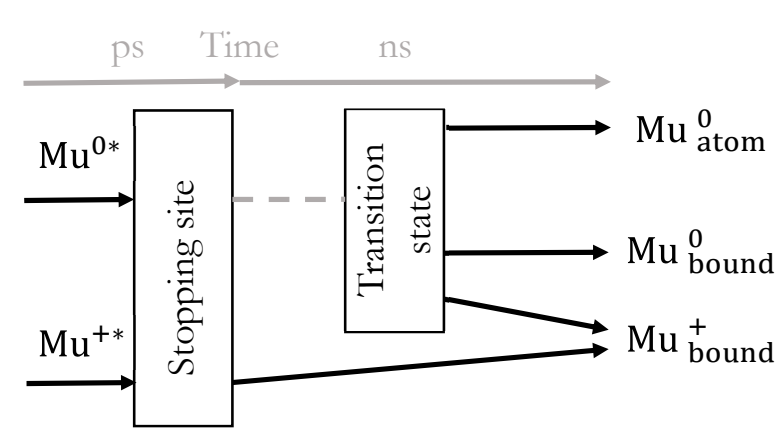

FIG. 2: Final step in muon implantation: Neutral $\left(\mathrm{Mu}^{0}\right)$ and charged $\left(\mathrm{Mu}^{+}\right)$muons come to rest at an interstitial site in the unrelaxed lattice (marked by $*$ ). $\mathrm{Mu}^{+}$forms directly the final bound configuration, but $\mathrm{Mu}^{0 *}$ may go through an intermediate (transition) state before the final configuration is formed.

teristics of the fast relaxing signal, i.e. prompt formation and relatively long lifetime (several ns to $\mu \mathrm{s}$ ), seems rather unrealistic.

Thus, the assignment of the fast relaxing signal to either $\mathrm{Mu}^{+}$or to $\mathrm{Mu}^{-}$has serious problems. We will show in the following that the assumption of neutral muonium $\mathrm{Mu}^{0}$ for this state is consistent with the experimental findings. The signal looks diamagnetic since the hyperfine interaction is weak and fluctuates, resulting in an almost zero average hyperfine splitting.

\section{THE TRANSITION STATE}

The incoming muons (or a part of them) likely capture an electron during the charge-exchange thermalization cycle and end up as neutral muonium in the unrelaxed lattice. This configuration is highly unstable and initiates a lattice relaxation to release the stress around the muonium. The final step in the implantation process is sketched in Fig. 2

Both $\mathrm{Mu}^{+}$and $\mathrm{Mu}^{0}$ stop in an excited configuration (indicated by an asterisk on the corresponding symbol in Fig. 2). The excitation is mainly due to the embedding into the unrelaxed lattice. $\mathrm{Mu}^{+}$reacts immediately with the lattice and forms a bound configuration. However, starting from $\mathrm{Mu}^{0}$ the formation of the final states may be delayed due to the necessity of a rearrangement of the lattice atoms. The duration of this transition situation (transition state in Fig. 2) may vary strongly from material to material and may depend on temperature and other external parameters (e.g. electrical field). If this time lies in the nanosecond region or below the transition state cannot by observed directly but may manifest itself by a phase shift and missing fraction. This feature was

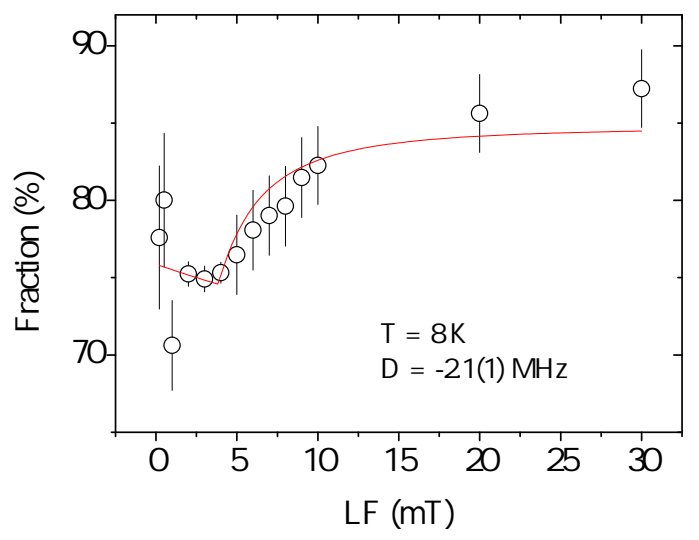

FIG. 3: $\mathrm{ZrO}_{2}: \mathrm{Mg}$ at $8 \mathrm{~K}$, decoupling curve in longitudinal magnetic field. The fraction of the non-relaxing signal (plus the slowly relaxing part which is not separated here) is represented. The fit with a dipolar-like hyperfine interaction (solid line) yields $D=-2.1(1) \mathrm{MHz}$.

used extensively in chemistry experiments to derive reaction constants. ${ }^{3}$ For even shorter transition times one speaks of prompt formation of the final states, as observed in the classical cases of $\mathrm{Si}$ and Ge. ${ }^{1}$ If the lifetime of the transition state falls into the time window of $\mu \mathrm{SR}$ method, the state can be observed directly. We identify the fast signal mentioned above with this situation. A long lifetime of the transition state is expected if the localization of the muonium electron proceeds via an energy barrier.

We suggest that the transition state is actually paramagnetic, but the hyperfine interaction is small and fluctuates leading to an almost vanishing average value. This makes it particularly difficult to detect and justifies that it may have been overlooked in past experiments. However, the paramagnetic character of the signal can be measured, as we will now demonstrate on the zirconia samples which were recently investigated at the ISIS Facility of the Rutherford Appleton Laboratory (United Kingdom) $\left[{ }^{6}\right.$

\section{A. Longitudinal field decoupling}

The hyperfine interaction causes a depolarization of the muon spin in longitudinal magnetic fields. If the field is strong enough to decouple the electron and muon spins then the muon spin polarization remains unaffected. In Fig. 3 we plot the fraction of muons that do not form the fast component, in order to highlight the corresponding recovery curve of the muon spin polarization. The fraction change seen in Fig. 3 equals the corresponding change of the fast relaxing signal which disappears in the same field range. Thus, the fraction change corresponds to the decoupling of the interaction in the fast relaxing signal. 
Strongly anisotropic muonium has two regions where the electron and muon spin become decoupled.20 The first decoupling occurs when the electron Zeeman energy becomes comparable to the hyperfine interaction. Assuming that the recovery in Fig. 3 is due to this effect, this would yield a hyperfine interaction in the order of $100 \mathrm{MHz}$. However, the shape of the recovery curve, in particular the kink at low fields, does not conform to the theoretical prediction, whereas such a feature is well known as a dip in the polarization recovery in case of an anisotropic interaction. ${ }^{20}$ This depolarization mechanism occurs at the $1-2$ level crossing 120121 This effect occurs at a much higher field or correspondingly at a lower interaction value for a given field than the usual decoupling and is observable only in case of an anisotropic interaction. The anisotropy determines the width of the depolarization peak and actually causes that the two levels avoid the crossing (avoided level crossing).

We analyzed the data with the avoided-level-crossing model presented in Refs. 20 and 21. The fit required a very small value for the isotropic (contact) part of the interaction; it was therefore set equal to zero in the final analysis. Assuming a pure dipolar interaction $\left(A=D\left(3 \cos ^{2} \theta-1\right)\right)$, the fit of the recovery curve (Fig. 3) yields for the dipolar part $D=-2.1(1) \mathrm{MHz}$. The cusp-like behavior at low fields is obtained only with a negative value of $D$. We note that a dominant negative component of the hyperfine interaction is observed also in the case of bond-centered muonium in $\mathrm{Si}$, Ge and diamond 200 A dipolar-like interaction is expected if the electron wave function is concentrated not at the muon site but at neighboring atoms some distance away from the muon. Actually, in a recent measurement on $\mathrm{TiO}_{2}$, a predominantly dipolar interaction of this order of magnitude $(D \sim 1 \mathrm{MHz})$ was found $!^{22}$

Thus, the hyperfine interaction in the transition state (in case of $\mathrm{ZrO}_{2}: \mathrm{Mg}$ ) is in the order of $2 \mathrm{MHz}$ and fluctuates, including sign changes. In the average, the interaction is close to zero.

\section{B. Longitudinal field relaxation}

Figure 4 shows the relaxation rate of the fast relaxing signal of the $\mathrm{Mg}$-doped zirconia in longitudinal field. The fits were performed with two exponentially decaying functions, a slow and a fast component. The slow component was first fitted to the data in the range between 3 and $20 \mu$ s and then fixed for the final analysis. The obtained values for the fast signal are in the order of $1 \mu \mathrm{s}^{-1}$ (Fig. (4). We note that the fraction of muons in the fast component becomes very small for fields close to $10 \mathrm{mT}$. The total non-fast fraction shown in Fig. 3 basically corresponds to the full observable muon spin polarization. This implies that for fields around and above $10 \mathrm{mT}$ the analysis of the fast component becomes increasingly difficult and the corresponding relaxation becomes harder

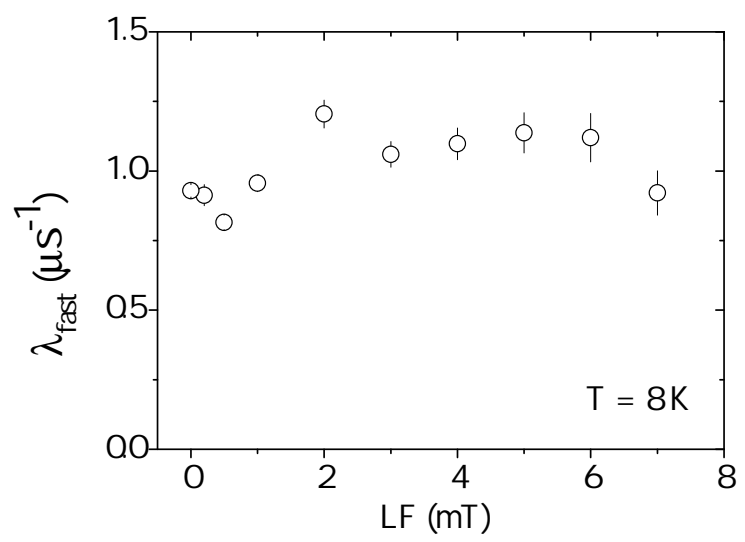

FIG. 4: Longitudinal-field relaxation rate of the fast relaxing signal in $\mathrm{ZrO}_{2}: \mathrm{Mg}$ at $8 \mathrm{~K}$.

to define. The values in Fig. 4 are therefore limited to $8 \mathrm{mT}$.

The relaxation rate of $1 \mu \mathrm{s}^{-1}$ is much too small to correspond to the frequency distribution expected from the hyperfine interaction measured in the decoupling experiment $(D \sim-2.1 \mathrm{MHz})$ if one assumes that the interaction is static. Dynamical narrowing must therefore take place.

The actually measured relaxation rate has an offset from lifetime broadening:

$$
\lambda_{\text {measured }}=\frac{1}{\tau}+\lambda_{\text {para }}
$$

where $\tau$ is the lifetime of the state and $\lambda_{\text {para }}$ is the static relaxation of the state. Thus the values in Fig. 4 constitute an upper limit of the paramagnetic relaxation rate.

For a rough estimate of the relaxation rate, we assume that the hyperfine interaction fluctuates between plus and minus values of a fixed interaction. This is equivalent to spin flips of the electron and also represents an approximation of the fluctuating dipolar interaction for electrons changing sites on a sphere around the muon. Assuming $A_{h f}= \pm 1 \mathrm{MHz}$ and $\lambda_{\text {para }} \leq 1 \mu \mathrm{s}^{-1}$ one obtains a spin-flip rate $\lambda_{S F} \sim\left(2 \pi A_{h f}\right)^{2} /\left(4 \lambda_{\text {para }}\right) \geqslant 10 \mu \mathrm{s}^{-1] 23}$ Note that this is only an estimate of the order of magnitude, taking into account the crude assumptions in the derivation.

\section{Frequency shift}

In our model, the fast relaxing signal is due to a paramagnetic state with a fluctuating hyperfine interaction. Due to the fast sign change, the hyperfine splitting is not observed but rather only the average of the two lines. This average value will coincide with the diamagnetic line only in the high-field (Paschen-Back) limit, where the hyperfine lines are symmetric around the diamagnetic line. 


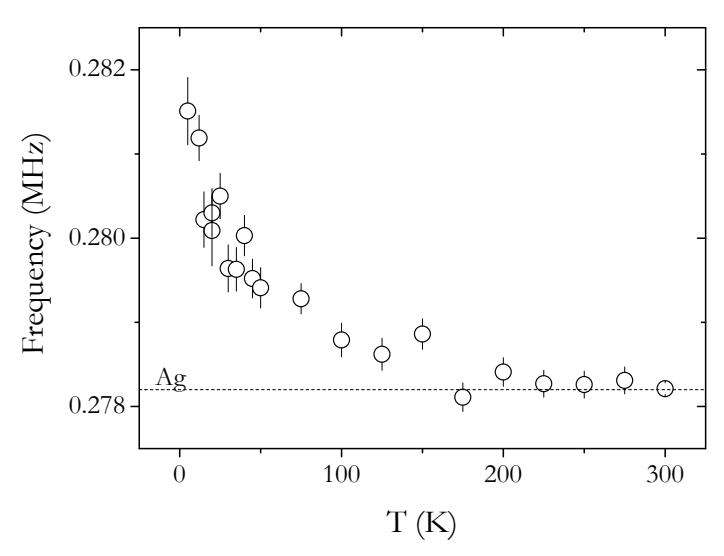

FIG. 5: $\mu$ SR frequency of $\mathrm{ZrO}_{2}: \mathrm{Mg}$ at $B=2 \mathrm{mT}$ as function of temperature. The expected frequency from the calibration with an Ag sample is shown for comparison.

However, if the external field approaches the transition region from the Paschen-Back to the Zeeman region the two lines are upward shifted and the average is displaced from the diamagnetic line. For the very small hyperfine interactions of the present case (in the order of $1 \mathrm{MHz}$ ) this effect can be observed only if the external field is in the order of $10 \mathrm{mT}$ or below. In these low-field measurements a separate evaluation of the frequency of the different components of the signal is difficult; we therefore show here the result of a fit with a common frequency of all components (Fig. 5).

Clearly an upward shift of the frequency compared to the calibration line is observed (Fig. 5). This shift vanishes above about $150 \mathrm{~K}$, at the temperature where the paramagnetic components of the signal disappear. This frequency shift is a clear indication of the presence of a paramagnetic interaction.

We conclude from the data analysis of the Mg-doped zirconia sample (which we consider as example for other cases) that the fast relaxing signal, in spite of its nearlydiamagnetic frequency behavior, corresponds to a paramagnetic state, i.e. to a muon with a nearby unpaired electron. The effective hyperfine interaction becomes very small due to fluctuations of the instantaneous hyperfine interaction (in the order of $\mathrm{MHz}$ ), assuming positive and negative values. The presence of the paramagnetic electron is evidenced by the decoupling behavior in low longitudinal fields and by the frequency shift in low transverse fields.

The precursor of the final muon states plays an important role in the $\mu \mathrm{SR}$ chemistry literature (see e.g. Refs. 3, 24 and the literature cited therein). In these studies the intermediate state is not observed directly but deduced from the missing fraction and the phase shift. In ref. 24 the controversial interpretation of the formation probabilities of the final states in the hot model and the spur model is discussed. Our interpretation is closer to the hot model, although differences exist due to the dif- ferent aggregate state of the material (solid compared to liquid or gaseous).

\section{Characteristics of the transition state}

The stopped muonium first forms a configuration similar to an effective-mass donor state in a dielectric medium. The formation of this configuration requires only a slight lattice rearrangement and may be formed almost promptly. We suggest that the transition state indicated in Fig. 2 has these donor state properties, i.e. a small electron binding energy (in the order of one to several $10 \mathrm{meV}$ ) and an extended electron wave function. The contact hyperfine interaction is very small, but the dipolar interaction from nearby electrons may be substantial (in the $\mathrm{MHz}$ range). An important difference to the usual donor state is, however, that the configuration is not static but fluctuates due to the local epithermal energy in the vicinity of the muon from the stopping process.

An adequate picture of the transition state is also to think of it as a large polaron, 25 weakly bound to the muon. The conversion to the final state corresponds then to the localization of the electron at a specific site in the lattice, i.e. the formation of a small polaron. This final step may in some cases lead to large energy gain which then contributes substantially to the total binding energy of the electron (self-trapping). Since the electron localization requires a lattice rearrangement, the process may be delayed and contribute to the delay of the conversion.

A donor-like weakly bound state has been introduced and extensively discussed in the work of Storchak et al ${ }^{15}$ These authors assume that the weakly bound state is formed by delayed capture of an electron from the ionization track. They assume that the muon stops as $\mathrm{Mu}^{+}$and exists for some time in this charge state until it captures the electron and forms neutral muonium. The electron capture ends the lifetime of the diamagnetic state. The justification of this model is largely based on electric field measurements which show that the state is easily ionized indicating weak binding of the electron. We note that the transition state described in the present paper (see Fig. 2 has the same weakly bound structure as in the Storchak model and therefore the electric field measurements do not distinguish between these models.

\section{THE BARRIER MODEL}

Why is there a delay in the formation of the final states? As mentioned above, the implanted muonium stops initially in the unrelaxed lattice. The final embedding of the muon into the lattice structure requires a rearrangement of the atoms in the neighborhood of the muon. The lattice relaxation may proceed via an energy barrier which delays the conversion. We note already here that this barrier is not the same as the barrier for the thermal 


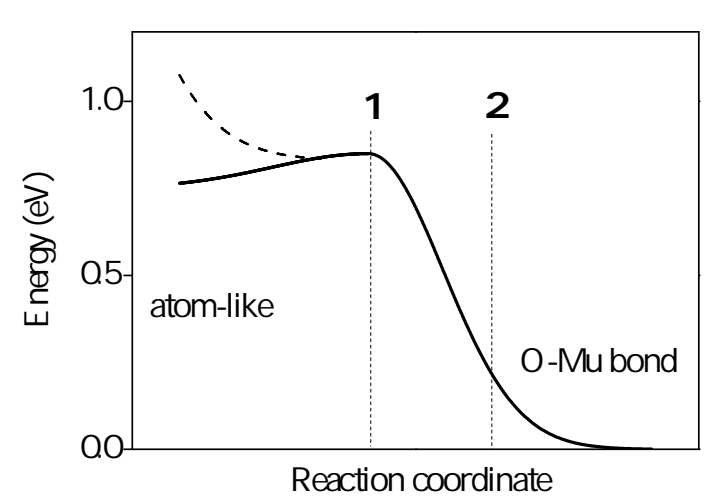

FIG. 6: Schematic representation of the total energy for the conversion from atom-like interstitial to anion-bound muonium (adapted from Ref. 26). The dashed line indicates the potential during the first steps of the lattice relaxation. The vertical lines (marked 1 and 2) indicate branching points (see text).

conversion from interstitial to bound muonium. The reason is that muonium has initially potential energy which is released during the relaxation process. We come back to that in more detail below.

Figure 6 shows schematically the total energy profile along the reaction coordinate for the jump of muonium from an interstitial site to a bound position (adapted from Ref. 26). We take this picture for a qualitative discussion of the conversion process.

The stopped muonium is initially in a similar configuration as the normal atom-like muonium (left of Fig. 6), but the potential energy is higher since the lattice is not relaxed (dashed line). Due to the stress, induced by the foreign particle, the lattice atoms start to move and readjust themselves to form the final state. The potential energy of muonium in the unrelaxed lattice and the local phonons from the muon stopping process can initiate jumps over the barrier even at low temperatures. This is a kind of "hot-atom" process.

Along the reaction path in Fig. 6, two critical points exist (indicated by vertical lines) which determine the formation probability of the final states (see discussion below).

\section{Branching point 1}

The maximum of the potential profile in Fig. 6 constitutes the threshold for the conversion. At the top of the profile (vertical line 1), muonium may return to the interstitial site and form atom-like muonium in the relaxed lattice, or it may continue the conversion to the bound configuration. The barrier height determines the ratio between atom-like interstitial muonium and anionbound muonium.

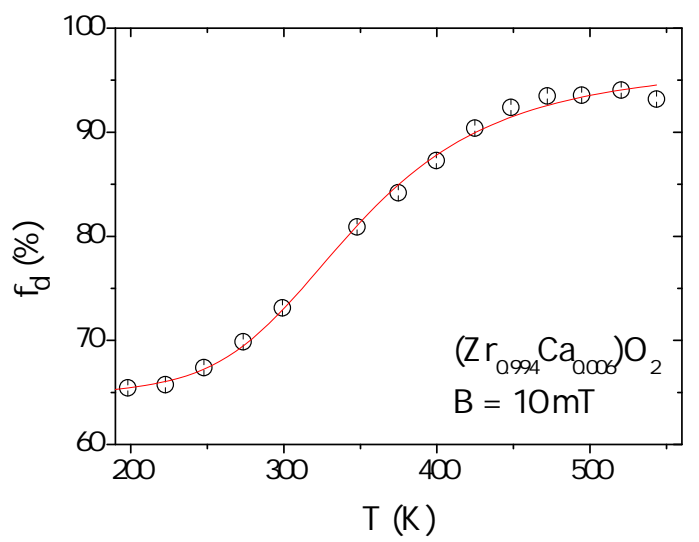

FIG. 7: Recovery of the diamagnetic fraction as a function of temperature for $\mathrm{ZrO}_{2}$ : $\mathrm{Ca}$ (from Ref. 6).

In the usual treatment of over-barrier hopping, a thermally activated process is assumed where the temperature provides the activation energy. In this model, the fraction of bound muonium is given by

$$
f_{\mathrm{b}}=f_{0} \frac{N \exp \left(-\frac{E_{b}}{k_{B} T}\right)}{1+N \exp \left(-\frac{E_{b}}{k_{B} T}\right)} .
$$

Here $f_{0}$ is the neutral fraction $\left(\mathrm{Mu}^{0}\right)$ of stopped muons, $N$ the statistical weight factor, $E_{b}$ the barrier height, $k_{B}$ the Boltzmann factor and $T$ the temperature. At low temperature, $T$ may be replaced by an effective temperature which accounts for the epithermal energy at the muon site.

The barrier height can be obtained from the thermal conversion from normal muonium to the bound muon configuration. As an example of the thermal conversion, the case of $\mathrm{ZrO}_{2}$ :Ca is shown in Fig. 7. Similar conversion curves were obtained for the other zirconia samples $\frac{6}{6}$ Barrier heights extracted from these data are given in table 【.

The data in table \ show a correlation between barrier height and the formation of a bound muon configuration: The higher the barrier, the fewer muons form a bound configuration. A quantitative evaluation of the correlation is difficult since the bound fraction contains also contributions from promptly reacting incoming $\mathrm{Mu}^{+}$. But the general tendency is obvious and is expected in the barrier model.

\section{Branching point 2}

The electron is only weakly bound in the transition state, and the binding energy fluctuates as the matrix atoms change positions. As the atom positions change and the total energy (of the muon and the lattice) 
TABLE I: Activation energy $\mathrm{E}_{a}$ derived from the thermal recovery of the diamagnetic fraction (see Fig. 7) for different zirconia samples. In the last column, the bound fraction $f_{b}$ (slowly relaxing component) at low-temperatures $(T \leq 10 \mathrm{~K})$ is given. We identify the activation energy with the barrier height and the bound fraction with the formation of a bound muon configuration. Data from Ref. 6].

\begin{tabular}{ccc}
\hline \hline Sample & $\begin{array}{c}\mathrm{E}_{a}(\mathrm{eV}) \\
\text { (barrier height) }\end{array}$ & $\begin{array}{c}f_{b}(\%) \\
\text { at } \mathrm{T} \leqslant 10 \mathrm{~K}\end{array}$ \\
\hline $\mathrm{ZrO}_{2}$ (undoped) & $0.26(2)$ & $37(2)$ \\
$\mathrm{ZrO}_{2}: \mathrm{Ca}$ (monoclinic) & $0.21(2)$ & $58(3)$ \\
YSZ & $0.12(2)$ & $83(2)$ \\
\hline \hline
\end{tabular}

changes as depicted approximately in Fig. 6, the binding energy of the electron to the muon may become extremely reduced. At some point (vertical line 2 in Fig. 6) this binding energy may eventually become nearly zero, as the electron energy level may come close to the conduction band. The electron may then be lost. The ionization terminates the lifetime of the transition state. We note that the electron loss may occur before the barrier height is reached (branching 2 before branching 1); in this case the barrier height does not determine the branching ratio between the atom-like and the bound muon configuration.

There is also the possibility that the electron remains bound till the end of the time spectrum or till the anionbound muonium is formed. This later process also ends the transition stage.

External fields weaken the electron binding in favour of ionization. Thus, the branching between neutral muonium and diamagnetic muon can be influenced, e.g. by the application of an external electric field. Electric field measurements by Storchak et al ${ }^{15}$ showed the existence of a weakly bound state in several semiconductor systems. These authors interpret these data as delayed muonium formation by capturing a radiolytic electron. However, the electron in the transition state described in the present work is also weakly bound and thus easily distractible by an external field. The difference to the weakly bound state proposed by Storchak et al. is that we assume that the electron is brought-in by the muonium formed in the charge exchange stage and not by delayed electron capture. A difference is also that we assume that the weakly bound state is not an excited electronic state (as in the model of Ref. 15) but an excitation of the lattice with the electron following adiabatically the changing atomic configuration.

\section{CONCLUSIONS}

We emphasize in this paper the importance of lattice relaxation for the formation of the final states in muon implantation. The muon is originally stopped in the pristine lattice. The formation of the final configuration requires a rearrangement of the nearby atoms. This process may take some time and lead to observable intermediate phenomena. We assign the so-called fast relaxating signal to this transition situation. The delay is caused by the duration of the relaxation process and is not due to the delayed capture or loss of an electron, as suggested in other publications (e.g. Refs. 7/9/15/27). The seemingly diamagnetic character of the fast relaxing signal is due to an almost vanishing average value of the fluctuating hyperfine interaction.

\section{ACKNOWLEDGEMENTS}

Apostolos Marinopoulos is thanked for helpful discussions. This work was supported with funds from (i) FEDER (Programa Operacional Factores de Competitividade COMPETE) and from FCT Fundação para a Ciência e Tecnologia under projects UID/FIS/04564/2016 and PTDC/FIS/102722/2008; (ii) $\mathrm{PhD}$ grant SFRH/BD/87343/2012 from FCT - Fundação para a Ciência e Tecnologia (RBLV).
* ruivilao@uc.pt

1 B. Patterson, Reviews of Modern Physics 60, 69 (1988).

2 S. F. J. Cox, R. L. Lichti, J. S. Lord, E. A. Davis, R. C. Vilão, J. M. Gil, T. D. Veal, and Y. G. Celebi, Physica Scripta 88, 068503 (2013).

3 M. Senba, D. J. Arseneau, J. J. Pan, and D. G. Fleming, Phys. Rev. A 74, 042708 (2006).

4 A. Weidinger, J. M. Gil, H. V. Alberto, R. C. Vilão, J. Piroto Duarte, N. Ayres de Campos, and S. F. J. Cox, Physica B: Condensed Matter 326, 124 (2003).

5 R. C. Vilão, J. M. Gil, A. Weidinger, H. V. Alberto, J. Piroto Duarte, N. Ayres de Campos, R. L. Lichti, K. H. Chow, and S. F. J. Cox, Nuclear Instruments and Methods in Physics Research Section A: Accelerators, Spectrometers, Detectors and Associated Equipment 580, 438 (2007).
${ }^{6}$ R. B. L. Vieira, R. C. Vilão, A. G. Marinopoulos, P. M. Gordo, J. A. Paixão, H. V. Alberto, J. M. Gil, A. Weidinger, R. L. Lichti, B. Baker, P. W. Mengyan, and J. S. Lord, Phys. Rev. B 94, 115207 (2016).

7 S. R. Kreitzman, R. F. Kiefl, D. R. Noakes, J. H. Brewer, and E. J. Ansaldo, Hyperfine Interactions 32, 521 (1986).

8 V. Storchak, J. H. Brewer, and G. D. Morris, Phys. Rev. B 56, 55 (1997).

9 J. D. Brewer, J. H. Brewer, G. D. Morris, D. G. Eshchenko, and V. G. Storchak, Physica B: Condensed Matter 289, 428 (2000).

10 S. F. J. Cox, J. L. Gavartin, J. S. Lord, S. P. Cottrell, J. M. Gil, H. V. Alberto, J. Piroto Duarte, R. C. Vilão, N. Ayres de Campos, D. J. Keeble, E. A. Davis, M. Charlton, and D. P. van der Werf, Journal of Physics: Condensed Matter 
18, 1079 (2006)

${ }^{11}$ I. Fan, K. H. Chow, B. Hitti, R. Scheuermann, A. I. Mansour, W. A. MacFarlane, B. E. Schultz, M. Egilmez, J. Jung, Y. G. Celebi, H. N. Bani-Salameh, B. R. Carroll, J. E. Vernon, and R. L. Lichti, Phys. Rev. B 78, 153203 (2008).

12 H. V. Alberto, R. C. Vilão, J. M. Gil, J. Piroto Duarte, R. B. L. Vieira, A. Weidinger, J. P. Leitão, A. F. Cunha, M. G. Sousa, J. P. Teixeira, P. A. Fernandes, P. M. P. Salomé, K. Timmo, M. Loorits, A. Amato, H. Luetkens, T. Prokscha, A. Suter, and Z. Salman, Journal of Physics: Conference Series 551, 012045 (2014).

13 R. B. L. Vieira, R. C. Vilão, H. V. Alberto, J. M. Gil, A. Weidinger, B. B. Baker, P. W. Mengyan, and R. L. Lichti, Journal of Physics: Conference Series 551, 012048 (2014).

14 R. C. Vilão, A. G. Marinopoulos, R. B. L. Vieira, A. Weidinger, H. V. Alberto, J. P. Duarte, J. M. Gil, J. S. Lord, and S. F. J. Cox, Phys. Rev. B 84, 045201 (2011).

15 V. G. Storchak, D. G. Eshchenko, and J. H. Brewer, Journal of Physics: Condensed Matter 16, S4761 (2004).

$16 \mathrm{H}$. Li and J. Robertson, Journal of Applied Physics 115, 203708 (2014).

17 K. H. Chow, B. Hitti, R. F. Kiefl, S. R. Dunsiger, R. L. Lichti,and T. L. Estle, Physical Review Letters 76, 3790 (1996).

18 R. C. Vilão, J. M. Gil, A. Weidinger, H. V. Alberto, J. Piroto Duarte, N. Ayres de Campos, R. L. Lichti, K. H.
Chow, S. P. Cottrell, and S. F. J. Cox, Physical Review B 77, 235212 (2008).

19 R. C. Vilão, J. M. Gil, A. Weidinger, H. V. Alberto, J. Piroto Duarte, and B. F. O. Costa, N. Ayres de Campos, R. L. Lichti, K. H. Chow, S. P. Cottrell, and S. F. J. Cox, Physica B: Condensed Matter 404, 888 (2009).

20 D. W. Cooke, M. Leon, M. A. Paciotti, P. F. Meier, S. F. J. Cox, E. A. Davis, T. L. Estle, B. Hitti, R. L. Lichti, C. Boekema, J. Lam, A. Morrobel-Sosa, and J. Oostens, Phys. Rev. B 50, 4391 (1994).

21 J. Piroto Duarte, Phd Thesis in Physics, University of Coimbra (2006), URL http://hdl.handle.net/10316/ 2533 .

${ }^{22}$ R. C. Vilão, R. B. L. Vieira, H. V. Alberto, J. M. Gil, A. Weidinger, R. L. Lichti, B. B. Baker, P. W. Mengyan, and J. S. Lord, Physical Review B 92, 081202 (2015).

23 V. G. Nosov and I. V. Yakovleva, Soviet Physics JETP 16, 1236 (1963).

24 D. C. Walker, S. Karolczak, H. A. Gillis, and G. B. Porter, Canadian Journal of Chemistry 81, 199 (2003).

25 J. T. Devreese, Polarons, Digital Encyclopedia of Applied Physics, edited by G. L. Trigg (Wiley, online) (2008), URL arXiv: cond-mat/0004497.

26 A. G. Marinopoulos, Physical Review B 86, 155144 (2012).

27 D. G. Eshchenko, V. G. Storchak and S. P. Cottrell, Physica B: Condensed Matter 404, 876 (2009). 